\title{
Taquicardia recíproca de la unión auriculoventricular: características electrofisiológicas en un paciente con la variedad paroxística. Caso clínico
}

\author{
Rodrigo Isa P, Rolando González A, Ismael Vergara S, \\ Mariana Baeza La. \\ Paroxysmal tachycardia in a patient \\ with a permanent form of junctional \\ reciprocating tachycardia
} \begin{abstract}
trant tachycardia whose anterograde conduction occurs via the His Purkinje and the retrograde conduction via an accessory pathway with slow conduction. The most common form is incessant tachycardia but a paroxysmal form also exists. We report a 35 years old female with recurrent paroxysmal tachycardia, that underwent electrophysiological evaluation. A left posterolateral accessory pathway was documented. Reciprocating paroxysmal tachycardia was induced by electrical stimulation and a successful pathway ablation was performed (Rev Méd Chile 2004; 132: 608-13).
\end{abstract}

Junctional reciprocating tachycardia is an atrioventricular reen-

(Key Words: Electrophysiology; Tachycardia, paroxysmal; Tachycardia, sinoatrial nodal reentry)

Recibido el 10 de octubre, 2003. Aceptado en versión corregida el 4 diciembre, 2003.

Laboratorio de Electrofisiología, Departamento de Enfermedades Cardiovasculares, Hospital Clínico y Facultad de Medicina, Pontificia Universidad Católica de Chile.

aEnfermera Universitaria

L a taquicardia paroxística supraventricular ـ(TPSV) es una variedad frecuente de arritmia que predomina en gente joven sin cardiopatía estructural. Sus mecanismos más frecuentes son la reentrada aurículo-ventricular (RAV) a través de un haz paraespecífico (HPE) y la reentrada nodal. Una variante poco frecuente de RAV, con una incidencia de $3 \%$, se produce a través de HPE caracterizado por tener conducción lenta ventriculoauricular (VA) sin con-

Correspondencia a: Dr. Rolando González A. Marcoleta 367, 2 piso. Teléfono: 3543176. Fax: 6327306.

E-mail: rgonzale@med.puc.cl ducción anterógrada ${ }^{1-4}$. En estos pacientes el electrocardiograma en ritmo sinusal es normal y en taquicardia el intervalo RP es mayor que el PR, con una onda P negativa en DII, DIII, aVF y V4 a V6.

Presentamos el caso de una mujer con episodios de TPSV por RAV con participación de un HPE con conducción lenta.

\section{CASO CĹNICO}

Mujer de 35 años de edad, sin antecedentes mórbidos relevantes y en capacidad funcional 
normal, con historia de seis meses de evolución de palpitaciones rápidas, regulares, sin relación a esfuerzo, de uno a diez minutos de duración. Inicialmente sus síntomas se presentaban cada dos semanas, y en los últimos dos meses, a diario. Consultó médico realizándose un ECG que resultó normal (Figura 1) y Holter de arritmias de $24 \mathrm{~h}$ que reveló episodios autolimitados de taquicardia de complejo angosto con una duración máxima de $15 \mathrm{~s}$ y frecuencia de hasta 150 latidos por min, compatible con TPSV. Se derivó a nuestro laboratorio para un estudio electrofisiológico (EEF).
EEF: Mediante punción venosa femoral derecha se introdujeron tres catéteres cuadripolares 6 French, que se ubicaron en la aurícula derecha alta, aurícula derecha septal a nivel del His y en el ápex del ventrículo derecho. Un catéter decapolar se avanzó a través de la vena yugular derecha hasta el seno coronario para registrar potenciales de aurícula izquierda. Se registraron intervalos basales y se realizó estimulación auricular y ventricular a frecuencia creciente y programada.

Los intervalos basales fueron normales, con un intervalo AH de 79 ms y HV de 38 ms (Figura 2).

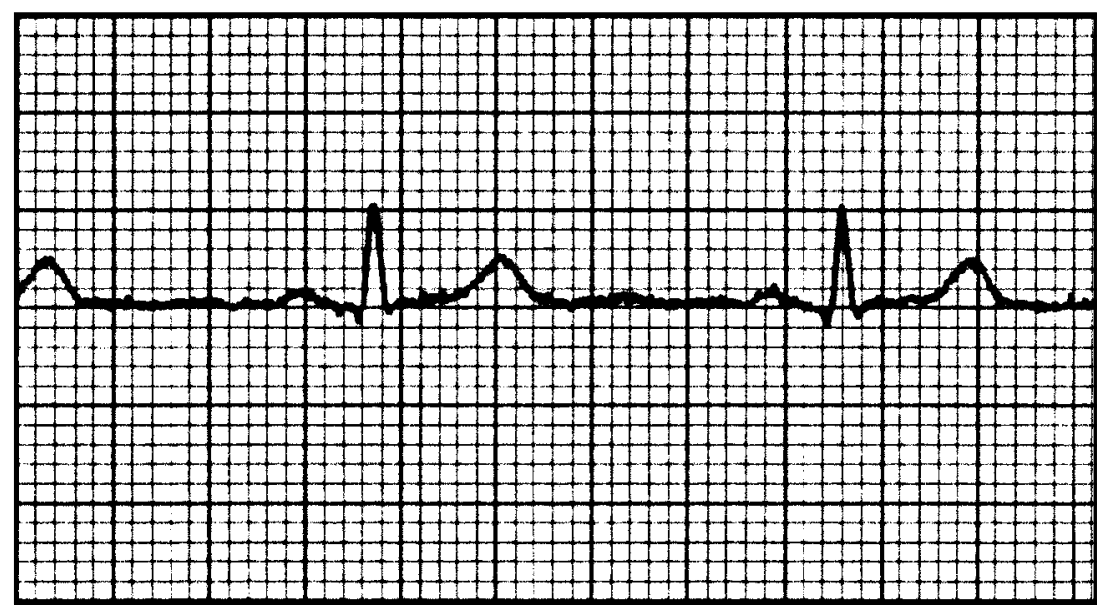

Figura 1. Derivación II del electrocardiograma de superficie: ritmo sinusal con frecuencia de 62 latidos por minuto e intervalo PR nor-

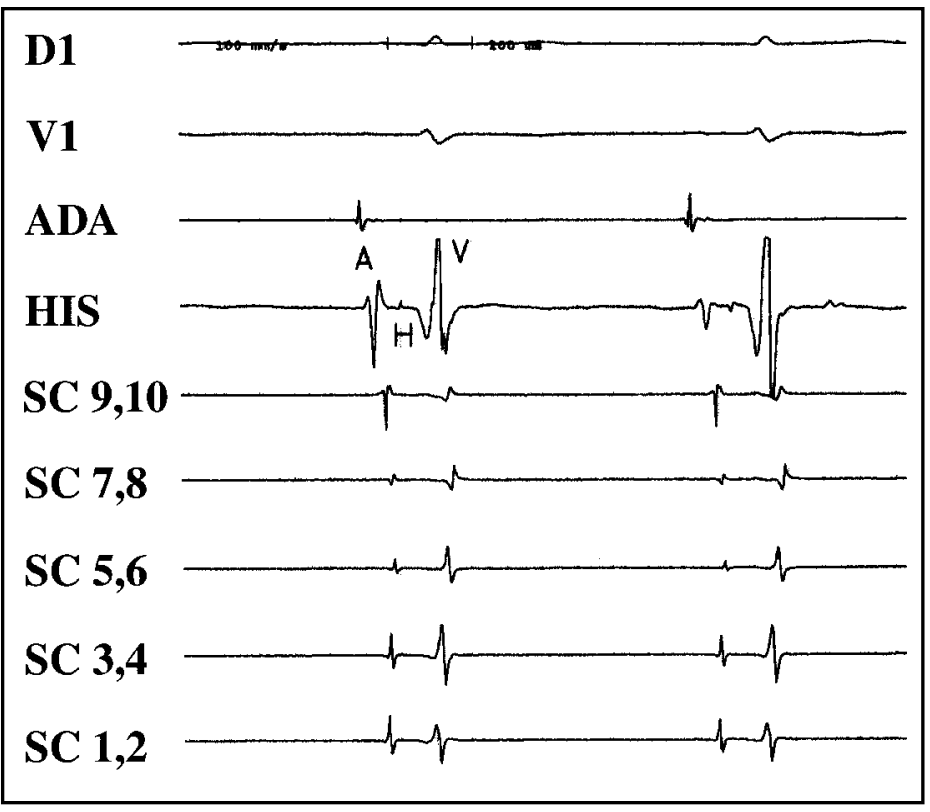

FIGURA 2. Registro simultáneo de electrocardiograma de superficie (D1 y V1) y electrogramas endocavitarios de aurícula derecha alta (ADA), aurícula septal (HIS) y seno coronario desde proximal hacia distal (SC 9,10 hasta 1,2). Durante ritmo sinusal los intervalos basales $\mathrm{AH}$ y HV son normales (79 y 38 ms respectivamente). 
En forma espontánea y mediante estimulación ventricular con frecuencia creciente y programada con extraestímulos acoplados a 460 ms y menos, se indujo taquicardia ortodrómica (Figura 3) con ciclo de $438 \mathrm{~ms}$, con activación auricular retrógrada más precoz en relación a los electrodos 3,4 del catéter en seno coronario. El intervalo VA, medido durante taquicardia, fue de $209 \mathrm{~ms}$ (Figura 4), el intervalo $\mathrm{AH}$ de $140 \mathrm{~ms}$ y el HA de $330 \mathrm{~ms}$, demostrándose la existencia de un haz oculto posterolateral izquierdo, con conducción lenta.

Por vía arterial femoral derecha se avanzó un catéter Cordis Webster 7 French con control de temperatura y por vía retroaórtica se procedió a aplicar pulsos de radiofrecuencia $(\mathrm{RF})$ a 50 Watts y $55^{\circ} \mathrm{C}$ durante taquicardia, en relación a la señal auricular más precoz a nivel del anillo mitral posterolateral, logrando la interrupción de la conducción por el HPE a los 6,8 s del segundo pulso de RF.

Post fulguración exitosa, se demostró una disociación VA y no se logró reinducir la taquicardia pese a la estimulación en condiciones basales y bajo isoproterenol $1 \mu \mathrm{g} / \mathrm{min}$, hasta $1 \mathrm{~h}$ de observación post RF (Figura 5).

\section{Discusión}

El diagnóstico diferencial de las TPSV con RP largo incluye las taquicardias auriculares, las reentradas nodales atípicas y las RAV por HPE con conducción lenta. En esta paciente, la secuencia de activación endocavitaria durante taquicardia confirmó la participación de un HPE.

La existencia de HPE con conducción retrógrada lenta y decremental fue descrita por Coumel en $1967^{5}$. Desde entonces, numerosas series han sido publicadas ilustrando las características clínicas, electrofisiológicas y los cambios en el tratamiento de esta arritmia.

La existencia de HPE con conducción retrógrada lenta y decremental genera el sustrato anatómico para una variedad especial de TPSV por RAV. En esta taquicardia participan las aurículas, el nodo auriculoventricular (AV), los ventrículos y el HPE, coexistiendo dos zonas de conducción lenta: por vía anterógrada el nodo $\mathrm{AV}$, y por vía retrógrada el HPE, generando una condición ideal para que esta taquiarritmia tenga un comportamiento incesante.

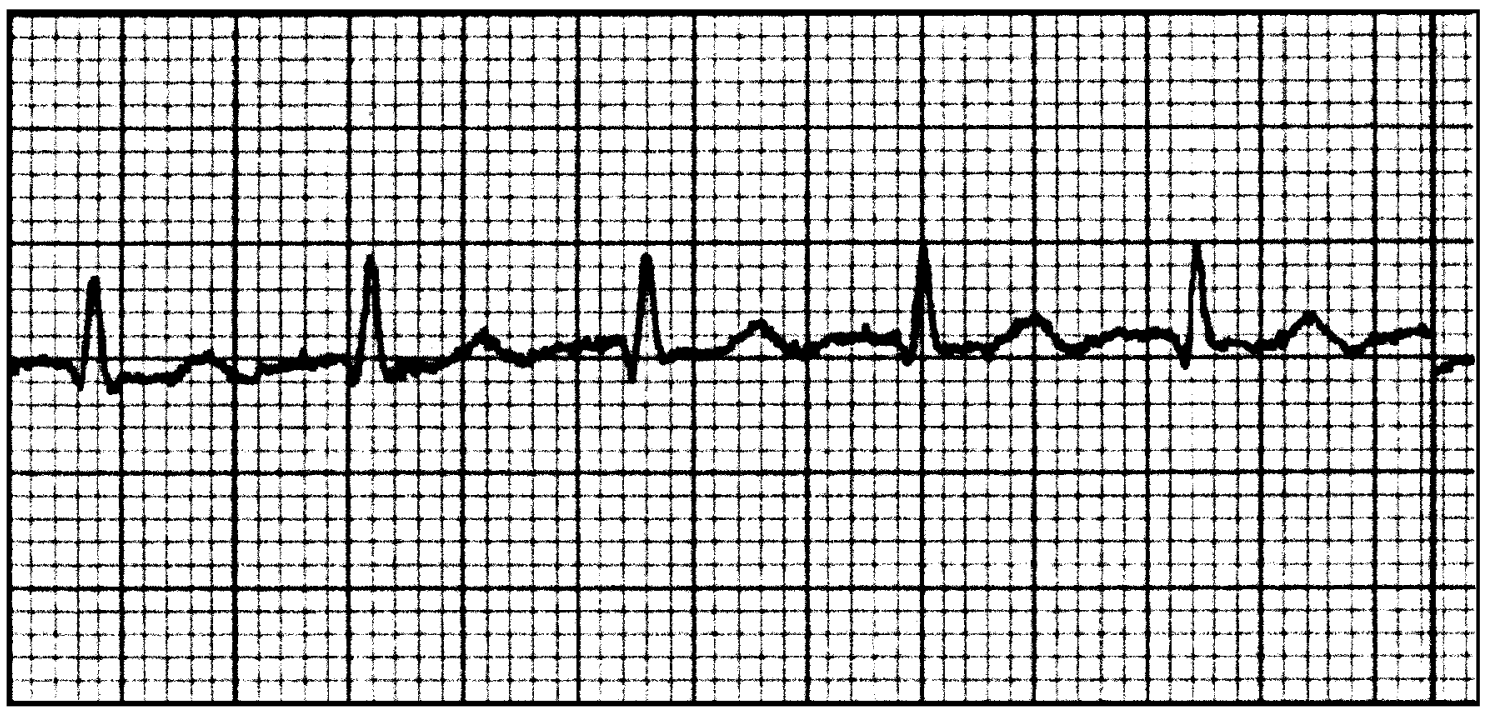

FIgURA 3. Derivación II del electrocardiograma de superficie durante taquicardia ortodrómica. Destaca un complejo QRS angosto, con onda P negativa después de la onda T e intervalo RP mayor que el PR. El ciclo de la taquicardia es de $438 \mathrm{~ms}$. 


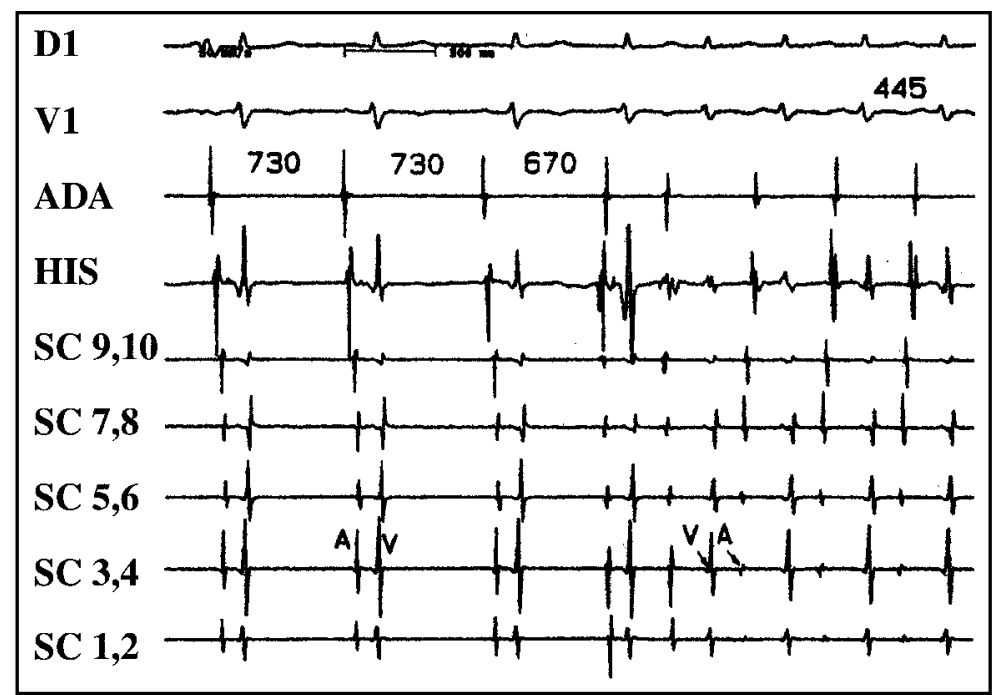

FIgURA 4. Registro simultáneo de electrocardiograma de superficie (D1 y V1) y electrogramas endocavitarios de aurícula derecha alta (ADA), aurícula septal (HIS) y seno coronario desde proximal hacia distal (SC 9,10 hasta 1,2) durante estimulación auricular programada. Con ciclo de estimulación auricular de 730 ms y extraestímulo acoplado a $670 \mathrm{~ms}$ se induce taquicardia ortodrómica con ciclo de $445 \mathrm{~ms}$. Se observa que la señal auricular más precoz se obtiene a nivel del SC 3,4. Además se observa que el intervalo VA (flechas) durante la taquicardia está prolongado (209 ms), demostrándose la existencia de un haz posterolateral izquierdo con conducción retrógrada lenta.

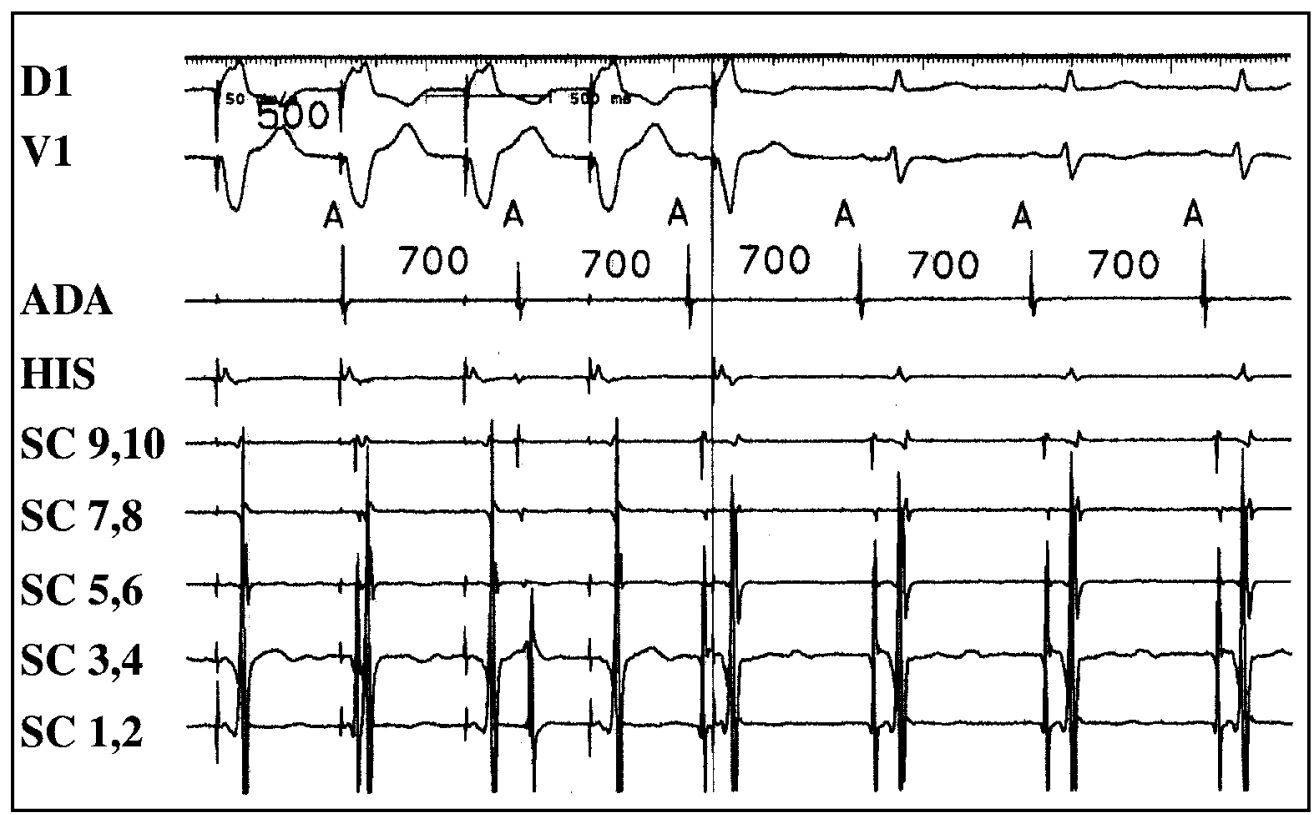

FigURA 5. Registro simultáneo de electrocardiograma de superficie (D1 y V1) y electrogramas endocavitarios de auńcula derecha alta (ADA), auńcula septal (HIS) y seno comnario desde proximal hacia distal (SC 9,10 hasta 1,2) durante estimulación ventricular con ciclo de $500 \mathrm{~ms}$, post fulguración con radiofrecuencia. Se observa captura ventricular con disociación ventrículo auricular (ciclo auricular espontáneo de 700 ms). Esto confima el éxito del procedimiento. 
El intervalo VA más precoz medido durante taquicardia o tras estimulación ventricular es el elemento clave para el diagnóstico de un HPE de conducción lenta. En nuestra paciente, el intervalo VA medido durante taquicardia fue de 209 ms, concordante con los datos de la literatura que reporta intervalos entre 160 y $420 \mathrm{~ms}$ para este tipo de HPE ${ }^{1-4,6}$. En nuestro laboratorio, la media de este intervalo en HPE no decrementales es de $80 \pm 6 \mathrm{~ms}$ (experiencia no publicada).

Histológicamente, estos HPE consisten en bandas de tejido fibromuscular atrial cuya conducción lenta y decremental ha sido atribuida a un curso tortuoso, con cambios en la resistencia axial $^{7}$. En forma alternativa, pero no mutuamente excluyente, se ha postulado que la conducción lenta y decremental de estas vías sea secundaria a depolarizaciones parciales de fascículos atriales anómalos. En este sentido, Lerman y $\mathrm{Col}^{8}$ estudiaron las diferentes propiedades electrofisiológicas de las vías retrógradas decrementales en las taquicardias con $\mathrm{RP}$ largo. Evaluaron la respuesta a adenosina y verapamilo en 5 pacientes con taquicardia recíproca de la unión (PJRT). Observaron al menos dos tipos de respuesta electrofisiológica: vías que sólo responden a adenosina, lo que sugiere que estarían parcialmente depolarizadas ( $>60 \mathrm{a}$ $-70 \mathrm{mV}$ ) vs aquellas que responden a ambos fármacos, lo que representa vías completamente despolarizadas, con potenciales de membrana en reposo de $-60 \mathrm{mV}$ o menos.

Las características electrofisiológicas de este tipo de taquicardia son dependientes de la edad de los pacientes afectados. Dorostkar y $\mathrm{col}^{9}$, en una serie que incluye pacientes pediátricos y adultos, observaron que la longitud del ciclo de la taquicardia aumenta significativamente con la edad de los pacientes. El ciclo aumentó de 308_64 $\mathrm{ms}$ en pacientes menores de 2 años, a $414+57 \mathrm{~ms}$ en pacientes entre 2 y 5 años, y a $445 \pm 57 \mathrm{~ms}$ en pacientes mayores de 5 años. Esto sería secundario a un enlentecimiento de la conducción retrógrada a través del HPE, lo que se refleja en un aumento del intervalo RP de 64\% (204+45 a $289 \pm 52 \mathrm{~ms}$ ) vs un aumento del intervalo PR de sólo $36 \%(109 \pm 25$ a $156 \pm 26 \mathrm{~ms})$, al comparar pacientes menores de dos años con mayores de cinco años.
La localización más frecuente de estos haces es posteroseptal derecha; sin embargo, diferentes autores han descrito localizaciones "atípicas" en las regiones posterolaterales derechas e izquierdas ${ }^{10}$, coexistencia con otros HPE sin conducción decremental, e incluso localizaciones múltiples en un mismo paciente. Shih y col ${ }^{11}$ reportaron tres pacientes con dos HPE decrementales; uno con dos HPE en la pared libre ventricular izquierda, otro con HPE a nivel de la pared libre y pared posterior del ventrículo derecho, y el último, con dos HPE posteroseptales separados por un centímetro. Nuestro grupo reportó 5 pacientes de los cuales tres tenían HPE posteroseptales derechos, uno posteroseptal izquierdo y uno posterolateral izquierdo6. La paciente actual presentó durante taquicardia una activación auricular retrógrada más precoz a nivel del electrodo 3,4 del seno coronario, lo que traduce la existencia de un HPE posterolateral izquierdo.

Existen dos formas de presentación clínica de esta taquicardia, la forma incesante y la paroxística, siendo más frecuente la primera. Ambas poseen diferentes características electrofisiológicas y distintas consecuencias clínicas.

Yagi y $\mathrm{col}^{12}$ en un estudio comparativo de 5 pacientes con la variedad paroxística vs 6 pacientes con la variedad incesante, demostraron la importancia del análisis de las características de conducción retrógrada de los haces decrementales en el diagnóstico diferencial de estas dos formas de taquicardia. Los primeros tenían un intervalo AH significativamente más largo, tanto en ritmo sinusal ( 95 a 135 vs 50 a $90 \mathrm{~ms}, \mathrm{p}<0,01$ ) como en taquicardia ( 90 a 120 vs 50 a $90 \mathrm{~ms}, \mathrm{p}<0,01$ ), un intervalo HA en taquicardia significativamente más corto ( $278 \pm 69$ vs $440 \pm 107 \mathrm{~ms}, \mathrm{p}<0,02$ ), lo que genera una relación $\mathrm{AH} / \mathrm{HA}$ mayor en la variedad paroxística $(0,41 \pm 0,11$ vs $0,16 \pm 0,04, p<0,01)$, y un intervalo VA más corto ( $229 \pm 61$ vs $386 \pm 112$, p $<0,02$ ). La corta duración del intervalo $\mathrm{AH}$ en taquicardia produciría un intervalo VA más largo, incrementando la estabilidad del circuito de reentrada y favoreciendo el comportamiento incesante. Nuestra paciente, que clínicamente se presentó con una forma paroxística de taquicardia recíproca, tenía en ritmo sinusal, a diferencia de las observaciones de Yagi, un intervalo AH de $79 \mathrm{~ms}$ $\mathrm{y}$, en concordancia con éste, un intervalo VA durante taquicardia de 209 ms y una relación AH/ HA 0,42 . 
Esta arritmia es usualmente refractaria al tratamiento antiarrítmico con drogas. Chien y $\mathrm{col}^{13}$ reportaron una serie de 6 pacientes catalogados como refractarios a múltiples terapias farmacológicas, entre ellas, quinidina, disopiramida, flecainida, digoxina, procainamida, beta bloqueadores, propafenona, amiodarona y verapamilo, en quienes se realizó un tratamiento ablativo endocavita-

\section{REFERENCIAS}

1. Aguinaga L, Brugada J, Anguera I, Mont LL, Valentino M, Eizmenfi I et al. Seguimiento a largo plazo de pacientes con la forma permanente de taquicardia recíproca de la unión tipo Coumel tratados mediante ablación con radiofrecuencia. Rev Esp Cardiol 1998; 51: 218-23.

2. Gaita F, Haissaguerre M, Giustetto C, Fischer B, Riccardi R, Scaglione M et al. Catheter ablation of permanent junctional reciprocating tachycardia with radiofrequency current. J Am Coll Cardiol 1995; 25: 648-54.

3. Shim-Ann CH, Ching T, Chem C, Shin W, Zu-Chi W, Chuen W ET aL. Electrophysiologic characteristics, electropharmacologic responses and radiofrequency ablation in patients with decremental accessory pathway. J Am Coll Cardiol 1996; 28: 732-7.

4. Oвel O, Cамm A. Accessory pathway reciprocating tachycardia. Eur Heart J 1998; suppl E: E13-24, E50-1.

5. Coumel P, Cabrol C, Fabiato A, Gourgon R, Slama R. Tachycardie permanent par rhythm reciproque. Arch Mal Coeur 1967; 60: 1830-64.

6. Acevedo M, Vergara I, Fajuri A, Cambón A, GonzÁlEZ R. Taquicardia incesante de la unión: características y rol de la fulguración en 5 casos. Rev Méd Chile 1995; 123: 833-40.

7. Critem G, Galagher J, Monda V, Coltorti F, SCHERILO M, Rossi I. Anatomic and electrophysiologic substrate of the permanent form of junctional reciprocating tachycardia. J Am Coll Cardiol 1984; 4: 606. rio. Además, estos pacientes están expuestos al riesgo de desarrollar una cardiomiopatía dilatada inducida por taquicardia cuando ésta adquiere un carácter incesante $^{14,15}$. Se han desarrollado numerosas formas de tratamiento no farmacológicas, pero en la actualidad se considera que la $\mathrm{RF}$ es el tratamiento de elección, con un éxito de 96 a $100 \% 1,6$ y una recurrencia de 13 a $17 \% 1,2,6$.

8. Lerman B, Greenberg M, Overholt E, Swerdlow C, Smith R, Seluers T et al. Differential electrophysiologic properties of decremental retrograde pathways in long RP' tachycardia. Circulation 1987; 76: 21-31.

9. Dorostkar P, Silka M, Morady F, Dick M. Clinical course of persistent junctional reciprocating tachycardia. J Am Coll Cardiol 1999; 33: 366-75.

10. Ticho B, Saul J, Hulse J, De W, Lulu J, Walsh E. Variable location of accessory pathways associated with the permanent form of junctional reciprocating tachycardia and confirmation with radiofrequency ablation. Am J Cardiol 1992; 70: 1559-64.

11. Shit H, Miles W, Kiein L, Hubbard J, Zipes D. Multiple accessory pathways in the permanent form of junctional reciprocating tachycardia. Am J Cardiol 1994; 73: 361-7.

12. Yagi $T$, Ito $M$, Odakura $H$, Namekawa A, Otoma J, IsHidA A. Electrophysiologic comparison between incessant and paroxysmal tachycardia in patients with permanent form of junctional reciprocating tachycardia. Am J Cardiol 1996; 78: 697-700.

13. Chien W, Cohen T, Lee M, Lesh M, Griffin J, SchiLeR $\mathrm{N}$ ET AL. Electrophysiologic findings and long term follow up of patients with the permanent form of junctional reciprocating tachycardia treated with catheter ablation. Circulation 1992; 85: 1329-36.

14. Packer D, Brady G, Worley S, Smith M, Cobb F, Coleman R et al. Tachycardia induced cardiomyopathy: A reversible form of left ventricular dysfunction. Am J Cardiol 1986; 57: 563-70.

15. Cruz F, Cheriex E, Smeets J, Atie J, Peres A, Pen O et AL. Reversibility of tachycardia induced cardiomyopathy after cure of incessant supraventricular tachycardia. J Am Coll Cardiol 1990; 16: 739-44. 